\title{
Two centuries of the equations of commutativity and associativity of exponentiation
}

\author{
LAJOS LÓcZI
}

\begin{abstract}
In this survey article we guide the reader through the solution of the commutative equation of exponentiation $x^{y}=y^{x}$ and that of the associative equation of exponentiation $x^{\left(y^{z}\right)}=\left(x^{y}\right)^{z}$. Various characterizations of the integer, rational, real and complex solutions are discussed together with some new results and open directions. The article is supplemented by a detailed and commented bibliography on the history of these equations.
\end{abstract}

Key words and phrases: commutativity of exponentiation, associativity of exponentiation, parametrizations of the solution set.

ZDM Subject Classification: F50, H30, I20.

\section{Introduction and notation}

The commutative equation of exponentiation possesses a long and rich history and has provided many of us with a fruitful source of challenging problems. As we will observe, several aspects of this equation have been discovered and rediscovered by many authors during the last two centuries. These results however often seem to be independent of each other or are scattered in the literature.

Our present discussion assembles the most noteworthy facts about the integer, rational, real and complex solutions of the equation

$$
x^{y}=y^{x}
$$

in Sections 2, 4 and 5 in order to enlighten the reader on this broad topic. Then, 
based on this commutative equation, positive integer, rational and real solutions of the associative equation

$$
x^{\left(y^{z}\right)}=\left(x^{y}\right)^{z}
$$

are derived in Section 3.

Finally, Section 6 comments on the results and known references collected in this survey and puts them into chronological order revealing some historical connections as well.

In order to keep the size of our exposition limited, known facts will only be stated, generally without repeating the proofs, though they are sometimes indicated to help the interested readers make their own discoveries.

In connection with (1), we will mainly focus on its nontrivial solutions, that is when $x \neq y$. Throughout the paper, $\operatorname{dom} f, \operatorname{ran} f$ and $f^{[-1]}$ denote the domain of definition, the range and the inverse function of $f$, respectively. In the context of multiple-valued powers, lr stands for the real, one-valued natural logarithm, with dom $\operatorname{lr}=(0,+\infty)$, moreover, $\arg z$ is the argument of the (complex) number $z$, with $\operatorname{ran} \arg =[0,2 \pi)$.

\section{Positive commutative pairs}

2.1. The positive real solutions of $x^{y}=y^{x}$

This section completely characterizes - via suitable parametrizations - all nontrivial positive real pairs $(x, y)$ satisfying $(1)$. Set

$$
\begin{aligned}
\psi(v) & :=v^{\frac{1}{v-1}} & & 0<v \neq 1, \\
\chi_{1}(u) & :=\left(1+\frac{1}{u}\right)^{u} & & u \in \mathbb{R} \backslash[-1,0], \\
\chi_{2}(u) & :=\left(1+\frac{1}{u}\right)^{u+1} & & u \in \mathbb{R} \backslash[-1,0],
\end{aligned}
$$

further, let $W$ denote the principal branch of the Lambert $W$-function with $\operatorname{dom} W=\left[-\frac{1}{e}, \infty\right)$ and $\operatorname{ran} W=[-1, \infty)$ defined by

$$
W(x) e^{W(x)}=x .
$$

(Therefore, the real-valued $W$-function is nothing else than the inverse function of $x \mapsto x e^{x}(x \geq-1)$. It can be used to represent solutions to a variety of 
transcendental equations. We remark that - as in the case of the logarithm and with suitable care - the $W$-function can be extended to the complex domain also.)

Proposition 2.1. All positive nontrivial real solutions to $x^{y}=y^{x}$ are given by any of the following formulae.

$$
\begin{array}{ll}
(x, y)=(\psi(v), v \psi(v)) & 0<v \neq 1 \\
(x, y)=\left(\psi(v), \psi\left(\frac{1}{v}\right)\right) & 0<v \neq 1 \\
(x, y)=\left(\chi_{1}(u), \chi_{2}(u)\right) & u \in \mathbb{R} \backslash[-1,0] \\
(x, y)=\left(x, x \psi^{[-1]}(x)\right) & 1<x \neq e .
\end{array}
$$

Alternatively, if $0<y<e<x$, one has

$$
(x, y)=\left(x,-\frac{x}{\ln x} W\left(-\frac{\ln x}{x}\right)\right) .
$$

Proof. By introducing a positive real parameter $v:=\frac{y}{x}>0$, equation (1) can be rewritten as $x^{v x}=(v x)^{x}$. Raising this to the power $\frac{1}{x}$, we get the crucial relation

$$
v x=x^{v} .
$$

$v=1$ (i.e. $x=y$ ) yields the trivial solutions. If $0<v \neq 1$, then $\ln v+\ln x=v \ln x$ hence $\ln x=\ln v^{\frac{1}{v-1}}$ thus $x=\psi(v)$ and $y=v x=v \psi(v)$, proving (3). A direct computation shows that $v \psi(v)=\psi\left(\frac{1}{v}\right)$, which justifies (4). As for (5), apply the substitution $u:=\frac{1}{v-1}$. (6) is simply obtained from (3) by using the definition of the inverse function $\psi^{[-1]}$. For $(7)$, analyse carefully the defining equations (together with the domains and ranges) of $\psi^{[-1]}$ and $W$ to prove the relation $W\left(-\frac{\ln x}{x}\right)=-\psi^{[-1]}(x) \cdot \ln x$ for $x>e$, then use (6). It can be easily verified that $(3)-(7)$ are solutions of (1) indeed.

Remark 1. If $0<v \neq 1$, then $\psi(v)$ is the number for which multiplication by $v$ or raising to the power $v$ amounts to the same thing, since $v \psi(v)=\psi(v)^{v}$.

Remark 2. According to (4), a solution $(x, y)$ is transformed into the solution $(y, x)$ by the substitution $v \mapsto \frac{1}{v}$. 
Remark 3. Symmetry of the solutions manifests itself in the fact, for example, that the function $x \psi^{[-1]}(x)$ is equal to its own inverse, in other words,

$$
x \psi^{[-1]}(x) \cdot \psi^{[-1]}\left(x \psi^{[-1]}(x)\right)=x
$$

holds true for $1<x \neq e$. (Deducing this property directly from the definition of $\psi^{[-1]}$ is instructive.) Symmetry also implies that the graphs of $\chi_{1}$ and $\chi_{2}$ are reflections of each other about the line $x=-\frac{1}{2}$ in the $x-y$ plane, that is $\chi_{1}(-u-1)=\chi_{2}(u)$ and $\chi_{2}(-u-1)=\chi_{1}(u)$.

Remark 4. Somewhat surprisingly, the upper left part of the nontrivial curve on Figure 1 can not be obtained from (7), since the right hand side of (7) gives trivial solutions for $x \in(0, e) \backslash\{1\}$, that is $-\frac{x}{\ln x} W\left(-\frac{\ln x}{x}\right)=x$ here.

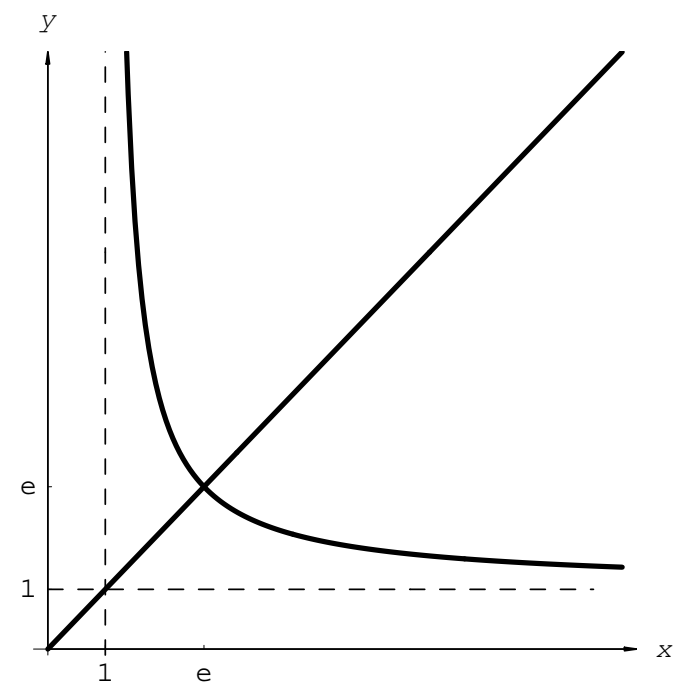

Figure 1. The graph of positive trivial and nontrivial solutions of $x^{y}=y^{x}$

Finally, we phrase some simple consequences.

Proposition 2.2. $x^{y}=y^{x}$ can not hold if $x \neq y$ and at least one of them is in $(0,1] \cup\{e\}$. Similarly, if $x, y>e$ and $x \neq y$, then $x^{y}=y^{x}$ does not admit any solutions. Moreover, if $x^{y} \leq e^{e}$ and $x \neq y$, then $x^{y} \neq y^{x}$. 
Proof. The first two statements can be deduced from Figure 1. To verify the third one, substitute (3) into $x^{y}$ to get

$$
\psi(v)^{v \psi(v)}=v^{\frac{v\left(\frac{v}{v-1}\right)}{v-1}},
$$

and prove that the range of this function is the interval $\left(e^{e},+\infty\right)$.

\subsection{Positive integer and rational solutions}

Now let us turn our attention to the nontrivial positive integer and positive rational solutions of $x^{y}=y^{x}$. A nice elementary number theoretical proof in the recent literature can be found in [30] or in [32]. We just repeat the main results here.

Proposition 2.3. All positive nontrivial rational solutions $(x, y)$ to $x^{y}=y^{x}$ are given by

$$
\begin{gathered}
(x, y)=\left(\left(1+\frac{1}{n}\right)^{n},\left(1+\frac{1}{n}\right)^{n+1}\right) \equiv\left(\chi_{1}(n), \chi_{2}(n)\right) \\
n \in \mathbb{Z} \backslash\{-1,0\},
\end{gathered}
$$

while the positive nontrivial integer solutions are only $(2,4)$ and $(4,2)$.

A curiosity about formula $\left(\chi_{1}(u), \chi_{2}(u)\right)$ will be highlighted in Section 6 .

\section{Positive associative triples}

In order to determine all positive real triples $(x, y, z)$ for which $\left(x^{y}\right)^{z}=x^{\left(y^{z}\right)}$ holds, notice that the left hand side is equal to $x^{y z}$. If $x \neq 1$, then we have $y z=y^{z}$. But this is the very equation we have encountered in the commutative case - see the proof of Proposition 2.1. If $z=1$, then every positive $y$ is a solution, otherwise $y=\psi(z)$. Therefore we have all positive real triples for which exponentiation is associative (see Figure 2):

$$
\begin{array}{ll}
(1, y, z) & \text { with } y, z>0, \\
(x, y, 1) & \text { with } x, y>0, x \neq 1, \\
(x, \psi(z), z) & \text { with } x, z>0, x \neq 1, z \neq 1 .
\end{array}
$$




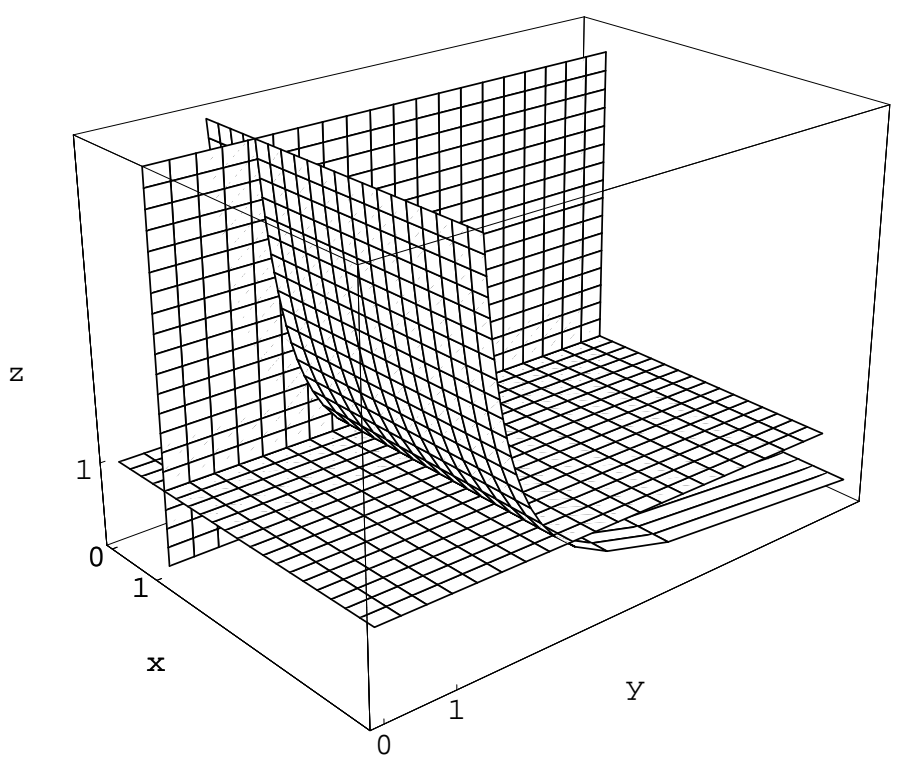

Figure 2

3.1. Positive integer and rational solutions

Proposition 3.1. Positive rational powers are associative if and only if the triple $(x, y, z)$ falls into one of the four categories below:

$$
\begin{array}{ll}
(1, y, z) & \text { with rational } y, z>0, \\
(x, y, 1) & \text { with rational } x, y>0, x \neq 1, \\
\left(x,\left(1+\frac{1}{n}\right)^{n}, \frac{n+1}{n}\right) \quad \text { with rational } 0<x \neq 1 \text { and } n \in \mathbb{N}^{+}, & \text {with rational } 0<x \neq 1 \text { and } n \in \mathbb{N}^{+} .
\end{array}
$$

Proof. The first two cases are trivial, while the other two follow from the formula $(x, \psi(z), z)$, since $z>0$ and $\psi(z)$ are rational by assumption, and comparing formulae (3) and (5), further using Proposition 2.3 yield that $\psi(z)=\chi_{1}(n)$ or $\psi(z)=\chi_{2}(n)$ must hold with some $n \in \mathbb{N}^{+}$, but $\psi$ is monotone decreasing, hence we have $z=\frac{n+1}{n}$ or $z=\frac{n}{n+1}$ for some $n \in \mathbb{N}^{+}$. 
Proposition 3.2. Positive integer solutions to (2) are given by the following triples:

$$
\begin{aligned}
& (1, y, z) \quad \text { with integers } y, z>0, \\
& (x, y, 1) \quad \text { with integers } x, y>0, x \neq 1, \\
& (x, 2,2) \quad \text { with integer } x>1 .
\end{aligned}
$$

\section{Real commutative pairs}

In order to extend the investigation of $x^{y}=y^{x}$ to $x, y \in \mathbb{R} \backslash\{0\}$, powers will be interpreted as multiple-valued complex expressions, i.e.

$$
x^{y}:=\left\{e^{y(\operatorname{lr}|x|+i \cdot \arg x+2 \pi k i)}: k \in \mathbb{Z}\right\} \subset \mathbb{C} .
$$

Definition 4.1. We say that a real pair $(x, y)(x, y \in \mathbb{R} \backslash\{0\})$ is a solution to $x^{y}=y^{x}$, if $x^{y} \cap y^{x} \neq \emptyset$.

It is shown in [10] that the set

$$
C:=\{(x, y): x, y \in \mathbb{R} \backslash\{0\}, x \neq y, x \operatorname{lr}|y|=y \operatorname{lr}|x|\}
$$

is the union of four curves $C_{j}(j=1,2,3,4)$, lying in the $j^{\text {th }}$ quadrant, see Figure 3 (though $C_{1}$ and $C_{3}$ have two points missing: $(e, e)$ and $(-e,-e)$, respectively). As we have seen, $C_{1}$ is a subset of the nontrivial solution set

$$
S:=\left\{(x, y): x, y \in \mathbb{R} \backslash\{0\}, x \neq y, x^{y} \cap y^{x} \neq \emptyset\right\}
$$

and an elementary argument shows in [10] that $S \subset C$. [21] proves that $S$ is countably dense on $C \backslash C_{1}$. However, it is important that $S \cap C_{j} \neq C_{j}(j=2,3,4)$. The necessary and sufficient condition for a real pair $(x, y)$ to be a nontrivial solution is given implicitly by the following proposition.

Proposition 4.2 (see [10], cf. [21]). $(x, y) \in S$ if and only if

$$
(x, y) \in C_{j} \quad(j \in\{1,2,3,4\})
$$




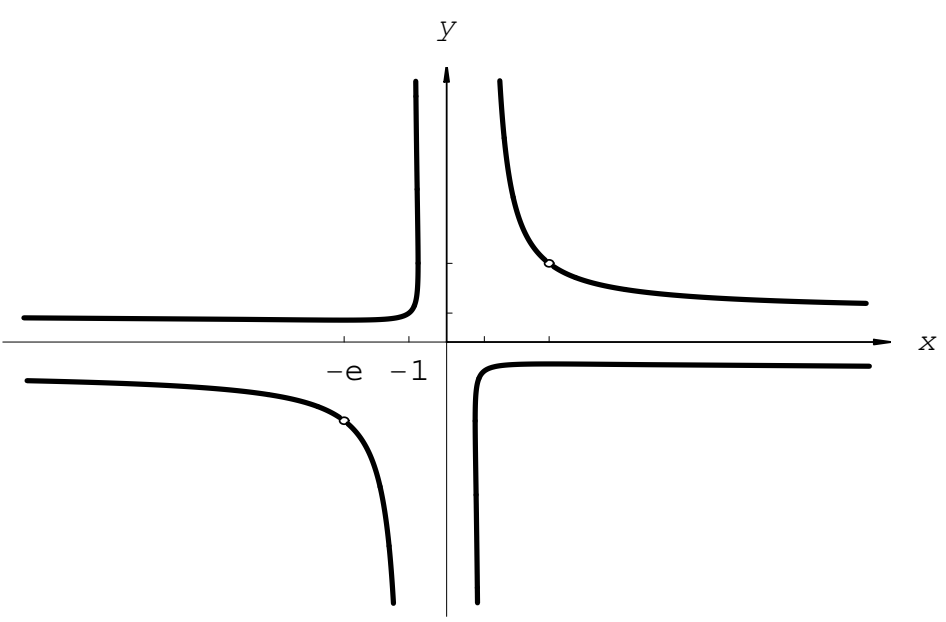

Figure 3. Not all points on the curves $C_{2}, C_{3}, C_{4}$ are solutions of $x^{y}=y^{x}$

and there exist integers $k, m, n \in \mathbb{Z}$ such that $(x, y)$ satisfies the $j^{\text {th }}$ equation below:

1. $k x=m y+n$

2. $2 k x=(2 m+1) y+2 n$

3. $(2 k+1) x=(2 m+1) y+2 n$

4. $(2 k+1) x=2 m y+2 n$.

Positive and negative rational solutions of (1) have also been described explicitly - in [22]. The interested reader can reconstruct them on one's own. There is, however, a subtle difference: while $C_{1} \cap(\mathbb{Q} \times \mathbb{Q}) \cap S=C_{1} \cap(\mathbb{Q} \times \mathbb{Q})$, this is no longer true for $C_{3}$, even though $C_{3}=-C_{1}$. (Propositions 2.3 and 4.2 help.) In contrast, $C_{2}$ and $C_{4}$ behave similarly, so the following proposition whose proof closely follows that of $(3)$ - suffices.

Proposition 4.3.

$$
\begin{aligned}
C_{4} & =\left\{\left(v^{-\frac{1}{v+1}},-v \cdot v^{-\frac{1}{v+1}}\right): 0<v\right\}, \\
C_{4} \cap(\mathbb{Q} \times \mathbb{Q}) & =\{(1,-1)\} .
\end{aligned}
$$


Corollary 4.4. If $(x, y) \in C_{4}$ and $x \in \mathbb{Q} \backslash\{1\}$, then $y \in \mathbb{R} \backslash \mathbb{Q}$.

Finally, note that the second sentence of Theorem 2 in [21] incorrectly states that if $(x, y) \in S \backslash C_{1}$ then $x$ and $y$ are both rational or both irrational. (In addition, there are other (typographical) errors in the counterpart of Proposition 4.2 in [21], but those do not affect the flaw in the proof of Theorem 2.) The proof in [21] misses the case $m=0$ in the fourth equation in Proposition 4.2, for example. A counterexample is $k:=0, m:=0, n:=1$, because then $x=2$ is rational, but the corresponding solution $y=-0.7666646 \ldots$ obtained from (9) must be irrational by Corollary 4.4. At the same time, this observation also answers affirmatively the open question in the same paper [21] by showing that there exist a rational number $x$ and an irrational number $y$ such that $(x, y) \in S$.

\section{Complex commutative pairs}

Now consider the equation

$$
z^{w}=w^{z}
$$

in the sense of Definition 4.1 with $z, w \in \mathbb{C} \backslash\{0\}$.

Proposition 5.1. $z^{w}=w^{z}$ is equivalent to demanding that there exist integers $k, l, m \in \mathbb{Z}$ such that

$$
\begin{gathered}
-|z| \cos \zeta \cdot \operatorname{lr}|w|+|w| \cos \omega \cdot \operatorname{lr}|z| \\
+(2 l \pi+\omega)|z| \sin \zeta-(2 k \pi+\zeta)|w| \sin \omega=0
\end{gathered}
$$

and

$$
\begin{gathered}
-|z| \sin \zeta \cdot \operatorname{lr}|w|+|w| \sin \omega \cdot \operatorname{lr}|z| \\
+(2 k \pi+\zeta)|w| \cos \omega-(2 l \pi+\omega)|z| \cos \zeta-2 m \pi=0,
\end{gathered}
$$

where $\zeta:=\arg z$ and $\omega:=\arg w$.

Proof. Use the definitions of multiple-valued powers, then take real and imaginary parts.

Due to the complex nature of these equations, no attempt is made here to give the general solutions of (11). However, existence can be established without much effort. 
Proposition 5.2. For every $w \in \mathbb{C} \backslash\{0,1\}$ equation (11) has a nontrivial solution.

Proof. The beginning of the proof is the same as in [2]. $z^{w}=w^{z}$ is equivalent to demanding that $\frac{\ln z}{z}=\frac{\ln w}{w}+\frac{2 \pi m i}{z w}$ holds for some $m \in \mathbb{Z}$. It is sufficient to take $m=0$ for now. Let $w \in \mathbb{C} \backslash\{0,1\}$ be arbitrary, and let $A:=\frac{\ln w}{w}$, for some value of the logarithm. (The original proof in [2] then went on by showing that the two polar curves defined by the real and imaginary parts of the equation $\frac{\ln z}{z}=A$ always intersect. More than a century later, we could give a different proof.) The exponential substitution $z=: e^{s}$ transforms the equation $\frac{\ln z}{z}=A$ into $s \cdot e^{-s}=A$. For $A=0$ (that is, for $w=1$ ), there are only finitely many solutions, namely $s=0$ (that is $z=1$ ). Hence, by virtue of Picard's theorem, $s \cdot e^{-s}=A$ must have infinitely many solutions for $A \neq 0$. It is seen that these have to be different modulo $2 \pi i$, thus $z=e^{s}$ possesses infinitely many different values. This proves that for any $w \in \mathbb{C} \backslash\{0,1\}$ there exists $z \in \mathbb{C} \backslash\{0,1\}, z \neq w$, such that $z^{w} \cap w^{z} \neq \emptyset$.

Remark 5. It is interesting to note that (11) has nontrivial solutions even for $w=1$. Indeed, suppose $z=r e^{i \varphi}$ with $r>0$ and $\varphi \in[0,2 \pi)$. Then $1^{z}=z^{1}$ is equivalent to $2 \pi k \cdot r \cos \varphi=\varphi+2 \pi l$ and $-2 \pi k \cdot r \sin \varphi=\operatorname{lr} r$, for some $k, l \in \mathbb{Z}$, which admits a solution $k=1, l=0, r=0.261739 \ldots, \varphi=0.952826 \ldots$, for example. Figure 4 shows some other solutions for different values of $k$ and $l$.

Now we construct infinitely many nontrivial solutions of $z^{w}=w^{z}$.

Proposition 5.3. For every $v \in \mathbb{C} \backslash\{0,1\}$ the complex analogue of (3) yields a nontrivial solution to (11).

PROOF. Use the principal value of the expression $v^{\frac{1}{v-1}}$.

Example 1. Let $v:=1+i$. Then $v^{\frac{1}{v-1}}=(1+i)^{-i}$ and (using approximate values) we have $z:=2.06287-0.745007 i \in(1+i)^{-i}$, and $w:=v z=2.80788+$ $1.31787 i \in(1+i)^{1-i}$. Further, one can show that $z^{w} \ni 14.298+0.886354 i \in w^{z}$ holds.

The analogy with formula (3) is not complete, however. Namely, we have shown there if $x, y>0$ and $x \neq y$ are given positive numbers such that $x^{y}=y^{x}$, then $x=v^{\frac{1}{v-1}}$ for $v:=\frac{y}{x}$. We have discovered (effectively using Proposition 5.1 


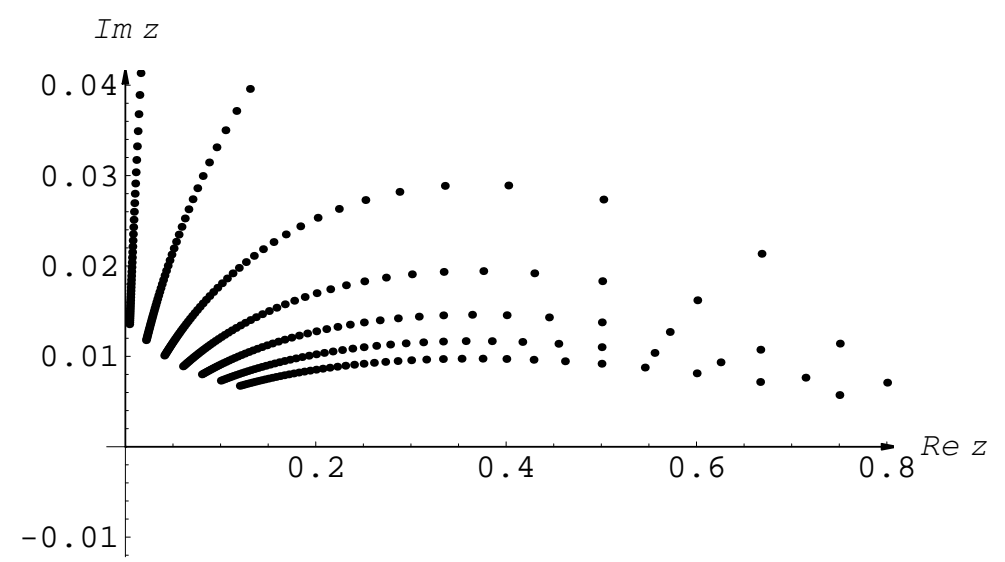

Figure 4. Some nontrivial solutions of $1^{z}=z^{1}$

and the computing power of Mathematica) that this is no longer true in the complex case.

Example 2 (Values are correct to 8-digit precision). There exist $z, w \in \mathbb{C} \backslash$ $\{0,1\}, z \neq w$ such that $z^{w} \cap w^{z} \neq \emptyset$, but $z \notin v^{\frac{1}{v-1}}$ for $v:=\frac{w}{z}$. Indeed, let

$$
z:=0.33300028-0.01584562 i \text { and } w:=0.66600056-0.03169125 i,
$$

then $v:=\frac{w}{z}=2$ and $z^{w} \ni-0.35447941+0.62145363 i \in w^{z}$, but clearly, $z \notin$ $v^{\frac{1}{v-1}}=\{2\}$.

Scrutinizing the operations in the proof of (3) reveals the explanation. The main point is that well-known identities in the positive reals are weakened in the complex case, for example, only $a^{b c} \subset\left(a^{b}\right)^{c}$ holds for general complex numbers.

Thus it is natural to ask finally whether every nontrivial solution of $z^{w}=w^{z}$ can be obtained from $\left(v^{\frac{1}{v-1}}, v \cdot v^{\frac{1}{v-1}}\right)$ by suitably choosing (possibly different) values of $v^{\frac{1}{v-1}}$ :

Open Problem. Is it true that given $z, w \in \mathbb{C} \backslash\{0\}$ arbitrarily such that $z \neq w$ and $z^{w} \cap w^{z} \neq \emptyset$ hold, there exist $v \in \mathbb{C} \backslash\{0,1\}$ and $z_{1} \in v^{\frac{1}{v-1}}, w_{1} \in v \cdot v^{\frac{1}{v-1}}$ such that $z_{1}=z$ and $w_{1}=w$ ? 


\section{Bibliographical remarks}

The body of the material brought together in our survey has been (re)discovered by the author (with the main exception being Section 4, which was motivated by [21] to settle a question and an incorrect statement contained therein and noticed lately by the present author). To be more explicit, we first indicate how our work is related to other papers.

Sections 2 and 3 can essentially be considered as the condensed version of our earlier article [30].

Later, an extensive search in the literature resulted in a gradually expanding collection of references concerning the commutative equation, see further remarks below. (We particularly recommend the relevant sections of the interesting survey [25] also containing a host of references. For the sake of completeness, we have quoted all of those regarding (1) explicitly.)

Nevertheless, it seems that there are still some results of ours which, to the best of our knowledge, were not published by other authors. These include (the already mentioned) Section 3 and Section 5 - with the exception of the content of Proposition 5.2 - as well as formula (7).

Finally, some brief comments and additional information on other references will follow.

Euler [1] himself mentions formulae (3) and (5). Figure 1 appears in his book among the analyses of transcendental curves and he also gives some positive rational solutions of (1) based on (5). [2] indicates that for every $w \in \mathbb{C} \backslash\{0\}$ there exist (in general infinitely many) $z \in \mathbb{C} \backslash\{0\}$ such that (11) holds. [3] contains formula (5). [4] gives a series expansion for the function $\psi^{[-1]}(x)$. [7] and [8] determines the positive integer and positive rational solutions of (1), respectively. [9] reports other references concerning (1) from the same era. [11] mentions a trigonometric parametrization of the real solutions of (1). [12] interprets the solution curve of (1) as an evolute. [13] determines the positive rational solutions as well. [14] formulates (3) and analyses the behaviour of those coordinate functions. Problems indicated in [15] discuss (3) and (5) from various perspectives, but no solutions to those exercises are given in the book.

[16] proves that all integer solutions of $(1)$ are $(2,4),(4,2)$ and $(-2,-4)$, $(-4,-2)$. [17] examines $(1)$ in algebraic extensions and in ideals.

Following [18] and [23], formula $\left(\chi_{1}(u), \chi_{2}(u)\right)$ reveals a wonderful and truly remarkable hierarchy:

- if $u \in \mathbb{R} \backslash[-1,0]$, it gives the positive real solutions of (1), 
- if $u \in \mathbb{Q} \backslash[-1,0]$, we get the solutions in the positive real algebraic field,

- if $\frac{1}{u} \in \mathbb{Z} \backslash[-1,0]$, then it yields solutions in the ring of positive algebraic integers,

- if $u \in \mathbb{Z} \backslash[-1,0]$, the formula gives the positive rational solutions,

- while if $u \in\{-2,1\}$, it yields the positive integer solutions of (1).

(The last - seemingly strange - case and the role of the set $[-1,0]$ become clear if one turns back to Remark 3 in Section 2.1 about the reflection symmetry of the graphs of $\chi_{1}$ and $\chi_{2}$.)

Positive rational solutions of (1) are included in [19] and also in [20]. (According to [20], equation (1) in the positive integers was among the problems of the Moscow Mathematical Olympiad in 1948.) [24] examines the associative property. [25] devotes one sentence to the solution of (2). Positive real solutions of the equation $a^{a}=b^{b}$ are determined by [28] (see [25] also), however this is trivial for us: perform the substitutions $a \mapsto \frac{1}{x}$ and $b \mapsto \frac{1}{y}$. (This way one obtains all positive rational solutions to the equation $a^{a}=b^{b}$ as well.)

[29] proposes various inequalities involving $x^{y}$ and $y^{x}$ (consult [25] again for further details). [31] mentions an interesting relation between the positive real solutions of (1) and the convergence of the "power-tower" sequence

$$
a_{1}(x):=x \quad \text { and } \quad a_{n+1}(x):=x^{a_{n}(x)},
$$

where $x \in \mathbb{R}^{+}$and $n \rightarrow+\infty$. This sequence also appears in [15], and a variant in [26]. Refer - of course - to [25] as well.

For a functional representation of the $W$-function and of $x^{\frac{1}{x}}=y^{\frac{1}{y}}$, see [33], the latter equation being intimately connected to (1), see e.g. [32].

ACKnowledgement. The author would like to thank the referees for their helpful remarks and suggestions about the paper.

\section{References}

[1] L. Euler, Introductio in analysin infinitorum, $2^{\text {nd }}$ book, Par. 519, German translation, Berlin, 1788.

[2] K. Schwering, Über die Wurzeln der Gleichung $x^{y}=y^{x}$, Zeitschr. für Math. Phys. 23 (1878), 339-343.

[3] M. Luxenberg, Über die Gleichung $x^{y}=y^{x}$, Arch. Math. Phys. 66 (1881), 332-334.

[4] R. D. Carmichael, Problem \#211: If $x=v^{\frac{1}{v-1}}$, what is the $f(x)$ such that $v=f(x)$ ?, Amer. Math. Monthly 13 (1906), 18, 72. 
[5] R. D. Carmichael, On a certain class of curves given by transcendental equations, Amer. Math. Monthly 13 (1906), 221-226.

[6] R. D. Carmichael, On certain transcendental functions defined by a symbolic equation, Amer. Math. Monthly 15 (1908), 78-83.

[7] C. Herbst, Bestimmung der ganzen Zahlen, für welche die Beziehung gilt $x^{y}=y^{x}$, Unterrichtsbl. f. Math. 15 (1909), 62-63.

[8] A. Flechsenhaar, Über die Gleichung $x^{y}=y^{x}$, Unterrichtsbl. f. Math. 17 (1911), $70-73$.

[9] R. Schimmack, Zur Gleichung $x^{y}=y^{x}$, Unterrichtsbl. f. Math. 18 (1912), 34-35.

[10] E. J. Moulton, The real function defined by $x^{y}=y^{x}$, Amer. Math. Monthly 23 (1916), 233-237.

[11] P. Franklin, Relating to the real locus defined by the equation $x^{y}=y^{x}$, Amer. Math. Monthly 24 (1917), 137.

[12] R. C. Archibald, Problems-Note no. 9., Amer. Math. Monthly 28 (1921), 141-143.

[13] H. L. Slobin, The solutions of $x^{y}=y^{x}, x>0, y>0, x \neq y$, and their graphical representation, Amer. Math. Monthly 38 (1931), 444-447.

[14] P. Carrese, Sull'equazione $x^{y}=y^{x}$, Boll. Un. Mat. Ital. (2) 3 (1941), 318-320.

[15] N. M. Gjunter and R. O. Kusmin, Problems of higher mathematics I., Gosudarstvennoe Izdatel'stvo Tekhniko-teoreticheskoy Literatury, Moscow, Leningrad, 1949, Problems 1001, 1151, 1706, 1917 and 2000 (in Russian).

[16] L. E. Bush, The 1960 William Lowell Putnam Mathematical Competition, Amer. Math. Monthly 68 (1961), 635.

[17] A. Hausner, Algebraic number fields and the Diophantine equation $m^{n}=n^{m}$, Amer. Math. Monthly 68 (1961), 856-861.

[18] K. Mahler, R. Breusch, et al., Problem 5101: Solutions of an old equation, Amer. Math. Monthly 70 (1963), p. 571 and 71 (1964) p. 564.

[19] W. Sierpiński, Elementary Theory of Numbers, (translated from Polish), Państwowe Wydawnictwo Naukowe, Warszawa, 1964, 106-107.

[20] D. O. Shklarsky, N. N. Chentzov and I. M. Yaglom, Problem 124, Selected problems and theorems of elementary mathematics I., $2^{\text {nd }}$ Russian edn., Geometricheskie otsenki i zadachi iz kombinatornoy geometrii, Nauka, Moscow, 1965.

[21] A. F. Beardon, The real solutions of $x^{y}=y^{x}$, Math. Mag. 39 (1966), 108-111.

[22] S. Hurwitz, On the rational solutions of $m^{n}=n^{m}$ with $m \neq n$, Amer. Math. Monthly 74 (1967), 298-300.

[23] D. Sato, Algebraic solution of $x^{y}=y^{x}(0<x<y)$, Proc. Amer. Math. Soc. 31 (1972), 316.

[24] M. Gardner, Mathematical games, Scientific American 228 (May 1973), p. 105, and (June 1973) p. 110.

[25] R. A. Knoebel, Exponentials reiterated, Amer. Math. Monthly 88 (1981), 235-252.

[26] F. Nakhli, A Transcendental Sequence and a Problem on Commutativity of Exponentiation of Real Numbers, Math. Mag. 62, no. 3 (1989), 185-190. 
[27] M. Sved, On the Rational Solutions of $x^{y}=y^{x}$, Math. Mag. 63, no. 1 (1990), 30-33.

[28] J. D. Bomberger, On the solutions of $a^{a}=b^{b}$, Pi Mu Epsilon J. 9, no. 9 (1993), $571-572$.

[29] Feng Qi, Lokenath Debnath, Inequalities for power-exponential functions, J. Inequal. Pure and Appl. Math. 1(2) (2000), Art. 15, http://jipam.vu.edu.au/.

[30] Lajos Lóczi, Commutativity and associativity of exponentiation, Mathematics and Physics Journal for Secondary Schools 50, no. 1 (2000), 7-16 (in Hungarian).

[31] Roman Sznajder and Kanchan Basnyat, Dynamics of a certain sequence of powers, Internat. J. Math. and Math. Sci. 24, no. 4 (2000), 283-288.

[32] Y. S. Kupitzand H. Martini, On the Equation $x^{y}=y^{x}$, Elem. Math. 55, no. 3 (2000), 95-101.

[33] Yunhi Cho and Kyunghwan Park, What is the Inverse Function of $y=x^{\frac{1}{x}}$, Amer. Math. Monthly 108 (2001), 963-966.

LAJOS LÓCZI

DEPARTMENT OF DIFFERENTIAL EQUATIONS

BUDAPEST UNIVERSITY OF TECHNOLOGY

H-1521 BUDAPEST

HUNGARY

E-mail: Iloczi@math.bme.hu

(Received May, 2003) 